\title{
Role of time scales and topology on the dynamics of complex networks
}

\author{
Kajari Gupta ${ }^{1}$ and G. Ambika1,2,a) \\ 1) Indian Institute of Science Education and Research (IISER) Pune, Pune-411008, \\ India \\ ${ }^{2)}$ Indian Institute of Science Education and Research (IISER) Tirupati, Tirupati-517507, \\ India
}

(Dated: 23 April 2019)

The interplay between time scales and structural properties of complex networks of nonlinear oscillators can generate many interesting phenomena, like amplitude death, cluster synchronization, frequency synchronization etc. We study the emergence of such phenomena and their transitions by considering a complex network of dynamical systems in which a fraction of systems evolves on a slower time scale on the network. We report the transition to amplitude death for the whole network and the scaling near the transitions as the connectivity pattern changes. We also discuss the suppression and recovery of oscillations and the cross over behavior as the number of slow systems increases. By considering a scale free network of systems with multiple time scales, we study the role of heterogeneity in link structure on dynamical properties and the consequent critical behaviors. In this case with hubs made slow, our main results are the escape time statistics for loss of complete synchrony as the slowness spreads on the network and the self-organization of the whole network to a new frequency synchronized state. Our results have potential applications in biological, physical, and engineering networks consisting of heterogeneous oscillators.

PACS numbers: 43.25.-x, 43.25.+y, 89.75.-k, 64.60.aq, 89.75.Fb, 05.45.Df

Keywords: Complex networks, Nonlinear systems, Multiple time scales, Frequency synchronization, Selforganization, Amplitude death

Complexity of real world systems are studied mainly in terms of the nonlinearity in the intrinsic dynamics of their sub systems and the complex interaction patterns among them. In such systems, the variability and heterogeneity of the interacting sub systems can add a further level of complexity. In this context heterogeneity arising from differing dynamical time scales offers several challenges and has applications in diverse fields, ranging from biology, economy, sociology to physics and engineering. In our work, we study the interesting cooperative dynamics in interacting nonlinear systems of differing time scales using the frame work of complex networks.

\section{INTRODUCTION}

Multiple-timescale phenomena are ubiquitous in $\mathrm{Na}$ ture and their in-depth understanding brings in several novel challenges. Some of the examples of such phenomena in real world systems are neuronal electrical activity ${ }^{1-3}$, hormonal regulation ${ }^{4}-7$, chemical reactions ${ }^{8-10}$, turbulent flows ${ }^{11-13}$ and population dynamics ${ }^{14-16}$ etc. Although there have been isolated studies addressing its various aspects, there are still many interesting questions that demand multidisciplinary approaches. Several modelling frameworks have

\footnotetext{
a) Electronic mail: g.ambika@iisertirupati.ac.in
}

proposed methods to understand dual time scale phenomena in single systems, like dynamical models for neuronal dynamics 17 . However, studies on collective behavior of connected systems that differ in their intrinsic time scales, are very minimal with many open questions. In this context, the framework of complex networks provides a promising tool to study nonlinear multiple time scale dynamics.

The emergence of synchronization in interacting dynamical units is important for the functionality of many systems and coupled oscillator networks are often studied to understand their dynamics 18,19 . Several types of synchronization phenomena like complete, phase and generalized synchronization $\underline{20} \underline{22}$, as well as cluster synchronization ${ }^{23.24}$ have been studied in various contexts. However, frequency synchronization is of recent interest and has relevance in many realistic situations ranging from neuronal systems to power grids $\frac{25-28}{2}$, where the individual oscillators can have non-identical natural frequencies.

The suppression of oscillations or amplitude death $29-35$ is also an emergent phenomenon that has interesting implications. In an assembly of coupled systems, amplitude death emerges mainly due to specific nature of coupling like transmission delay, processing delay, dynamic coupling, nonlinear coupling, environmental coupling, etc. or due to parameter mismatch $33,34,36-43$ or time scale diversity 44.45 . Most often, amplitude death is required to suppress unwanted oscillations in connected systems.

In this context we note that the revival of oscillations is an equally important and related emergent phenomena in coupled oscillators. Often in many systems recovering oscillations from suppressed state is necessary 
for their proper functioning, to maintain the output intensity in arrays of power generators, to get maximum output from coupled laser systems even with transmission delay, to sustain oscillations in interacting cardiac cells etc $\underline{25}, \underline{48}$. oscillations from quenched state in coupled systems have been reported $51-55$.

Interestingly, heterogeneity of interacting systems plays an important role in the diversity and organization in many complex systems $56-59$. The dynamical processes underlying their complexity often display phase transitions and analysis and prediction of such transitions are crucial for their proper functioning. Heterogeneity can arise from the pattern of interactions or nature of connectivity among the systems. Thus, in scale-free networks, the heterogeneity is mostly from the broad distribution of node degrees such that there exists a few nodes with very high degrees. In addition, heterogeneity due to different dynamical time scales is prevalent in many complex systems. It is shown that functional hierarchy emerges through a form of self-organization of two distinct types of neurons, with multiple timescales and hence not only the spatial connections between neurons but also the timescales of neural activity are important in the mechanisms leading to functional hierarchy in neural systems $\underline{60}$. So also, widely different time scales are common in systems of chemical reactions. In a recent study in biochemical networks, the fastest time scales is shown to correspond to the chemical equilibrium between metabolites while the slower ones relate to more physiologically relevant transformations 61 . This brings out the need to analyse correlations between metabolites considering the characteristic time scales of the network. The heterogeneity in network connectivity is shown to result in frequency synchronized clusters in non-identical phase oscillators 62 .

The main motivation of the present study is to analyze the onset and characterization of interesting collective dynamics or emergent behaviour in a network of connected systems with differing dynamical time scales. Thus the heterogeneity in dynamical time scales that we introduce is different from earlier studies where connected systems with nonidentical intrinsic frequencies are considered. In two coupled slow and fast systems and in minimal networks of three or four systems, onset amplitude death and occurrence of frequency synchronized states with tunable emergent frequencies are studied recently 46,47 . By considering two types of complex networks, we study the interplay between heterogeneity in link structure or connectivity among the systems and their differing time scales that can lead to interesting collective behaviour. Our study primarily uses standard periodic oscillators of Rössler and Landau Stuart type so that it brings out the amplitude variations and their cross over behaviours as the slowness factor increases.

We find the difference in time scales and the heterogeneity in connectivity together can drive the whole network to frequency synchronized clusters. Increasing the heterogeneity in time scales by increasing the number of slow systems or the mismatch in time scales, the whole network settles to a state of no oscillations. The transitions to that state as well as recovery to slower oscillations with cross over in amplitudes are some of the interesting results of the study.

We also address the important question of what happens if part or even one node of a scale free network of systems suddenly slows down and then how does it affect the performance of the whole network. In this case, the robustness of the network to such changes is studied in terms of the time taken for each node to escape from the synchronized state leading the whole network to desynchronized dynamics. We find this phenomenon of loss of synchrony settles in a time that decreases with the degree of the node that becomes slow first. The desynchronization transition that happens over a characteristic time is followed by the reorganisation of the whole network to a frequency synchronized state and this self-organization time depends on the difference in time scales. In addition, we study the transition to amplitude death on a scale free network, with multiple time scales, drawn from a normal distribution and with time scales decided by the connectivity of the systems.

\section{RANDOM NETWORKS OF SLOW AND FAST PERIODIC SYSTEMS}

We construct a random network of $\mathrm{N}$ nodes where each node represents an $n$ dimensional dynamical system. The dynamical equation of each node is taken as

$$
\dot{X}_{i}=\tau_{i} F\left(X_{i}\right)+G \epsilon \tau_{i} \sum_{j=1}^{N} A_{i j}\left(X_{j}-X_{i}\right)
$$

where $\mathrm{i}=1,2, \ldots .$. N. $F\left(X_{i}\right)$ represents the intrinsic dynamics of each node. $A_{i j}$ represents the adjacency matrix of connections in the network with its elements having values 1 , if the nodes $i$ and $j$ are connected and zero otherwise. The topological connectivity of the random network is defined by a parameter $p$, where $p$ is the probability with which any two nodes of the network are connected. $\mathrm{G}$ is $\operatorname{diag}(1,0, \ldots .$.$) since we consider diffusive$ coupling between the first variables of the systems. The systems on the network are identical in their intrinsic dynamics except that they evolve at different time scales, $t_{i}$, which are scaled as $t_{i}=\tau_{i} t$ to get the equation[1. Then $\tau_{i}$ will function as a parameter defining the dynamical time scale of evolution of the $i^{\text {th }}$ system. We vary $\tau_{i}$ in the range $(0.1,1)$ to introduce time scale diversity in the network of systems.

Choosing periodic Rössler system as nodal dynamics, 
the equations that govern the dynamics are

$$
\begin{aligned}
& \dot{x}_{i}=\tau_{i}\left(-y_{i}-z_{i}\right)+\tau_{i} \epsilon \sum_{j=1}^{N} A_{i j}\left(x_{j}-x_{i}\right) \\
& \dot{y}_{i}=\tau_{i}\left(x_{i}+a y_{i}\right) \\
& \dot{z}_{i}=\tau_{i}\left(b+z_{i}\left(x_{i}-c\right)\right)
\end{aligned}
$$

With the parameters chosen as $a=0.1, b=0.1$ and $c=4$, the intrinsic nodal dynamics is periodic.

First, we consider the case of dual time scales, such that in the network, out of $\mathrm{N}$ identical systems, $m$ evolve on a slower time scale. The subset of oscillators with slower time scale is taken as S. Thus $\tau_{i}=\tau$ if i belongs to the set $\mathrm{S}$ and $\tau_{i}=1$ for other nodes. Then the value of $\tau$ indicates the extend of mismatch in time scales between the two sets of oscillators, smaller values of $\tau$ corresponding to larger mismatch. We analyse how the slowness of $m$ of the systems can affect the dynamics of the whole network. For this the system of equations in eqn.(2) are integrated using Adams-Moulton-Bashforth method, with time step 0.01 for 100,000 times and the last 10,000 values of the $\mathrm{x}$-variables are used for calculations in the study.

\section{A. Suppression and recovery of oscillations}

In this section, we report the general results on the coupled dynamics of the systems by varying the parameters involved, time scale of slow systems $(\tau)$, number of slow systems $(m)$ and coupling strength of connections $(\epsilon)$, keeping the probability of connection of the network $p=0.5$. We find that for sufficient time scale mismatch between slow and fast subsets of systems, for strong coupling, for a range $m$, all the systems go to a synchronized fixed point. This state is generally known as amplitude death(AD) $\underline{29}-\underline{35}$ in the context of coupled dynamics. The Fig. 1] shows the state of amplitude death in the random network of slow and fast systems.

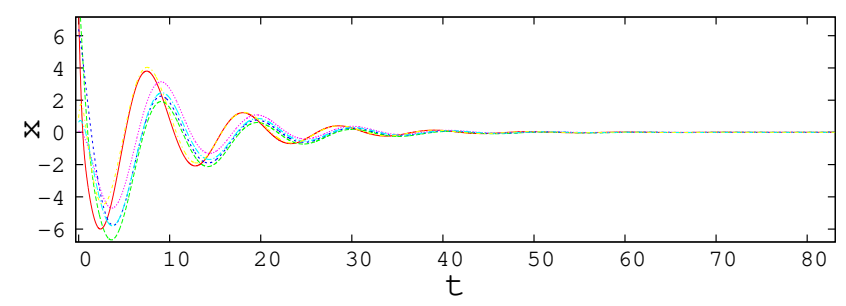

FIG. 1. (colour online) Time series of $x$ variables of periodic Rössler systems in a random network of slow and fast systems showing amplitude death state for $m=50, p=0.5, \tau=0.35$, $\epsilon=0.05$.

We calculate the difference between global maximum to global minimum, $A_{d i f f}$, from the time series of each oscillator. This averaged over all the $\mathrm{N}$ systems in the network serves as an index to identify onset of $\mathrm{AD}$ in the network, since $<A_{\text {diff }}>=0$ would correspond to $\mathrm{AD}$ state in the whole network. Using this we identify the region for occurrence of $\mathrm{AD}$ for different $m$, the number of slow systems present in the network, with suitable values chosen for the other parameters, $p, \tau, \epsilon$. We plot this region for two sets of values of $\tau$ and $\epsilon$ in Fig. 2, This shows

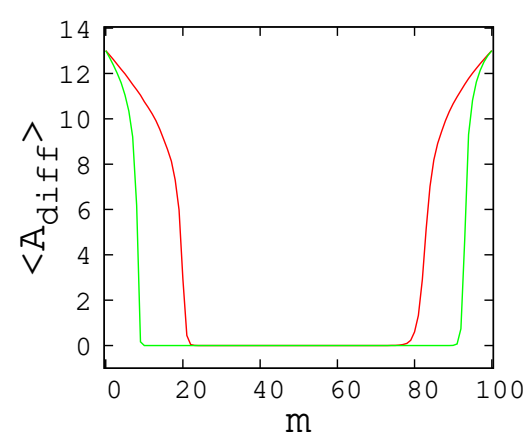

FIG. 2. (colour online)Variation of average $A_{\text {diff } f}$ with $m$. Here $\tau=0.35$ and $\epsilon=0.01$ for red curve and $\tau=0.35$ and $\epsilon=0.05$ for green curve. $\mathrm{N}=100, p=0.5$.

that a minimum number of slow systems is required for $\mathrm{AD}$ to occur, denoted as $m_{1}$. As $m$ increases the heterogeneity decreases and the network recovers from $\mathrm{AD}$ state beyond a certain value, $m_{2}$. Thus, suppression of dynamics happens as $m$ reaches a critical minimum value $m_{1}$ and recovery to oscillatory state happens beyond the second critical value, $m_{2}$; both these values depend on other parameters like $\tau, p$ and $\epsilon$ of the system.

With $m$ chosen from the region of $\mathrm{AD}$ in Fig. 2, we isolate the region of $\mathrm{AD}$ in $(\tau, \epsilon)$ plane, for a chosen $p=$ 0.5, and this is shown as region 1 in Fig. 3 .

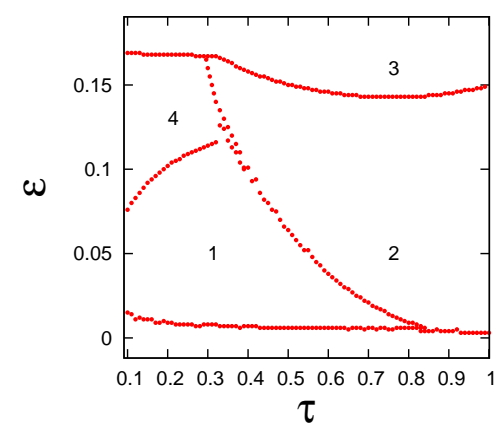

FIG. 3. (colour online)Regions of different dynamical states in the parameter plane $(\tau, \epsilon)$. Region 1 corresponds to $\mathrm{AD}$, 2 corresponds to frequency synchronization, 3 leads to instability and 4 is a transient state where systems start diverging from fixed point state before reaching unstable state. Here $m=50, p=0.5, \mathrm{~N}=100$. 


\section{B. Frequency synchronized dynamics}

In this section we present the possible dynamical states outside the region of $\mathrm{AD}$ in the parameter plane $(\tau, \epsilon)$. When coupling is strong and time scale mismatch is small, all the systems in the network settle to an organized state with oscillations of differing amplitudes but same frequency. This state of frequency synchronization is seen in region 2 in the Fig. 3. This is identified by calculating the frequency of each oscillator from its $\mathrm{x}$-time series using equation,

$$
\omega=\frac{1}{K} \sum_{k=1}^{K} \frac{2 \pi}{\left(t_{k+1}-t_{k}\right)}
$$

where $t_{k}$ is the time of the $k^{t h}$ zero crossing point in the time series of the oscillator and $K$ is the total number of intervals for which the zero crossings are counted. In this state, the oscillations of slow systems are relatively closer in phase and so are fast oscillators among themselves but the phase difference between slow and fast sets is relatively large. Below region 2, with low coupling strength the oscillators show a two-frequency state and as time scale mismatch increases they become periodic with two separate time scales. For very high coupling strength in the region marked as 3, network becomes unstable but before this there is region for low $\tau$ (region 4 ), where the systems are in a transient state, diverging from AD (Fig. 3).

\section{Crossover phenomena in the emergent dynamics for large $m$}

When the systems are in the state of frequency synchronized oscillations, corresponding to region 2 in Fig. 3. the amplitudes of slow and fast sets of systems vary from each other. In general, for low $m$, we observe that amplitudes of slow systems are smaller than those of fast systems, while for higher $m$ this behavior gets reversed with the slow set having larger amplitudes than the fast one. Thus, we observe a novel phenomenon of crossover behavior in the amplitudes as $m$ is varied. To show this explicitly, we study the average amplitude of all the slow systems and that of all the fast systems as $m$ is varied keeping the values of $\tau$ and $\epsilon$ in frequency synchronization state. We find that at a critical value of $m$, the amplitude of slow and fast systems undergoes a reversal as shown in Fig. 4

When $\mathrm{m}=0$ or $\mathrm{N}$, the systems are all completely synchronized. For other values of $\mathrm{m}$, there is approximate synchronization among systems in each set, slow and fast, but are phase shifted between them. Then the number of systems included in the coupling that come with mismatch or the number of systems from the other set, is effectively deciding the amplitude of oscillations. Thus for small $\mathrm{m}$, the slow systems have smaller amplitude due to

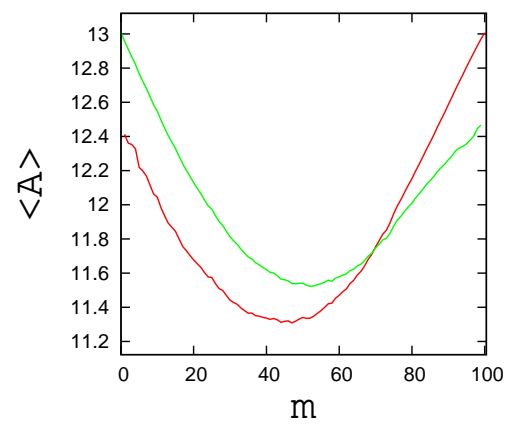

FIG. 4. (colour online) Crossover in magnitudes of amplitudes of slow and fast sets of oscillators, in the random network of 100 systems. Average amplitude of slow set is shown in (red) and that of fast in green. Here $p=0.5, \tau=0.7, \epsilon=0.05$.

the larger number of phase shifted and mismatched systems from the fast set. This gets reversed as $m$ becomes large and close to N. At a certain value of $m$, the cross over point, the effect of mismatched and phase shifted terms in the coupling have equal effects for both sets.

In the state of frequency synchronization, we also observe a similar cross over in the synchronized frequency, which is high for low $m$ and very low for high $m$. The synchronized frequency calculated using eqn.(3) for all the oscillators is plotted as function of $m$ in Fig. 5. For each particular $\tau$ the mean frequency of the intrinsic slow and fast frequencies is also shown (black lines). Thus, when the synchronized frequency for any given $\tau$ crosses the mean and decreases below that with larger $m$, we say frequency suppression occurs. The value of $m$ for which this happens is noted as the crossover point for the emergent frequency of the oscillators.

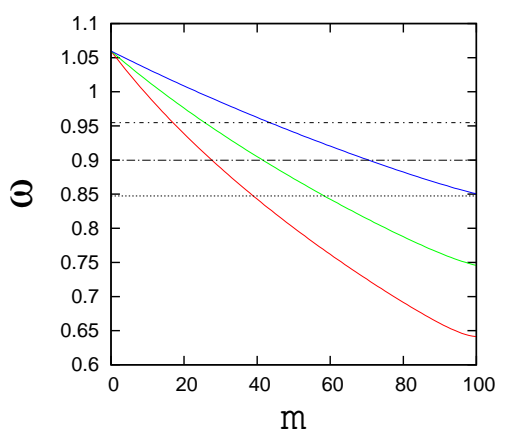

FIG. 5. (colour online) Frequency vs $m$ for $\epsilon=0.05$ $\tau=0.6$ (red), 0.7 (green) and 0.8(blue) showing crossover to frequency suppressed state as the emergent frequency crosses the mean value of fast and slow frequencies shown by corresponding black lines(increasing order of $\tau$ from below to above) . $p=0.5$. 


\section{Transition to amplitude death and connectivity of the network}

The topology or structure of the random network used, depends on its connectivity which is decided by the probability of connections $p$. As $p$ is increased from 0 to 1 , the topology goes from sparsely connected to fully connected network. In order to understand the role of topology or the connectivity of the network in the transition to $\mathrm{AD}$, we study the collective behavior of all the systems by varying $p$, for an $m$ value that lies in the AD region of Fig. 2] and values of $\tau$ and $\epsilon$ from the AD region in the parameter plane $(\mathrm{Fig} 3)$. For each value of $p$, we take 100 realizations of the network and check what fraction of them goes to AD state for the whole network. This fraction of the realizations $f$ gives the probability of transitions. We plot $f$ for different values of $m$, to get the corresponding transition curves. We observe that as $m$ increases, the transition to $\mathrm{AD}$ occurs at lower values of $p$, till it reaches a minimum and with further increase of $m$, the transitions move to higher values of $p$. This is clear from Fig 6. The threshold value for the transition, where half of the realizations go to amplitude death, is taken as $p_{t}$.

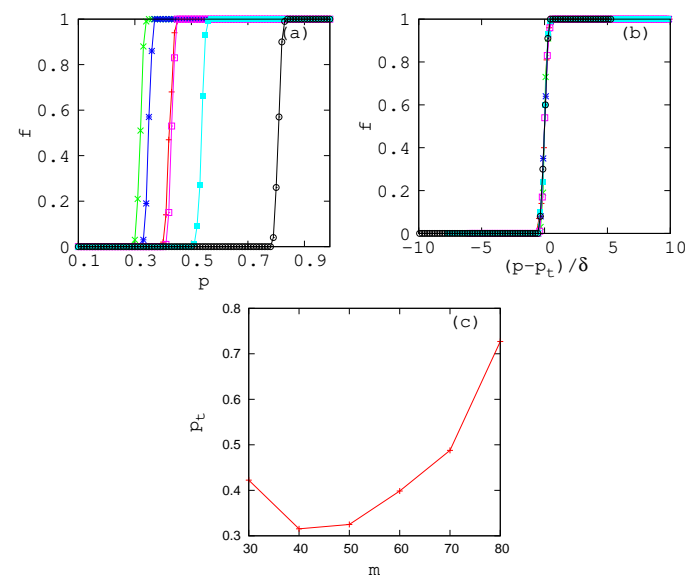

FIG. 6. a)(colour online)a)Fraction of realizations $f$ for the transition to $\mathrm{AD}$, plotted with the probability $p$ of connections in the network, b) normalized transition curves for $m=30$ (red, plus), 40(green, cross), 50(blue, star), 60(magenta, square), 70(cyan, solid square), 80(black, circle) and c) Variation of critical $p$ value, $p_{t}$ with $m$. Here $\tau=0.35$ and $\epsilon=0.01$.

For each value of $m$ used, we get the width of the transition curve as $\delta$ and normalize the transition curves by replacing $p$ with $\left(p-p_{t}\right) / \delta$. Then we find all the transition curves fall on top of each other revealing a universal behavior. This data crunched curve is shown in the Fig. 6(b). Moreover, the threshold value $p_{t}$ varies with $m$ as shown in Fig. 6)(c), which indicates a minimum at a particular value of $m$. At this value of $m$, the heterogeneity in the network is optimized or maximized and hence transition to AD can happen even with sparsely connected network corresponding to minimum value of p, the probability of connections in the network. For the random network of 100 periodic Rössler systems, $p_{t}$ is observed to be minimum when the number of slow systems is around 40 .

\section{Scaling with size of network}

We repeat the above analysis and obtain the transition curves for different network sizes, with $\mathrm{N}=100,150,200$, $300,500,600$, keeping $m / \mathrm{N}$ ratio fixed at 0.5 . We notice the larger the size of the network lower the value of $p$ at which transition takes place. To study the scaling properties of these transitions, with system size, we fit each transition curve with the functional form

$$
f=\left(p-p_{c}\right)^{\alpha}
$$

Here the value of $p_{c}$ is chosen as the one where the function gives best fit, and then the corresponding value of the scaling index $\alpha$ is calculated for each transition curve. Our results indicate that the index $\alpha$ varies with the network size N. To get the value of $\alpha$ in the large size limit, i.e. as $\mathrm{N}$ approaches infinity, we plot the calculated $\alpha$ vs $1 / \mathrm{N}$ and take the asymptote as $1 / \mathrm{N}$ goes to zero. This comes out to be 0.68 , which within numerical errors, can be taken as $2 / 3$. (Fig. 7)

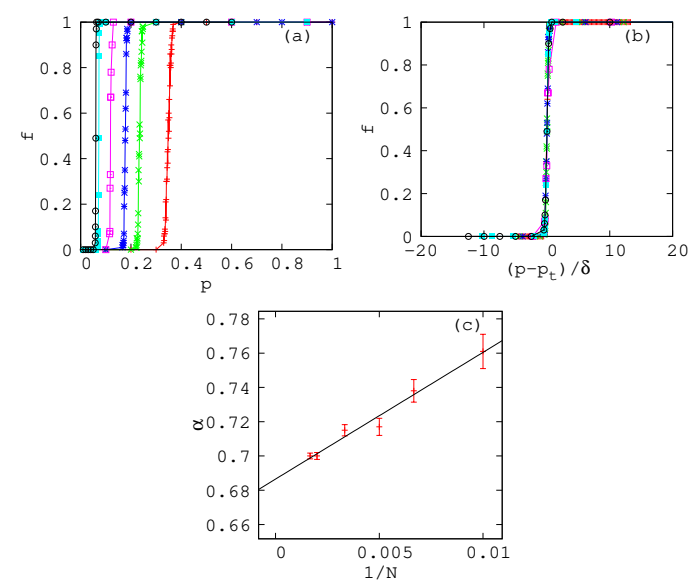

FIG. 7. (colour online)Fraction of realizations $f$ for the transition to $\mathrm{AD}$ plotted with the probability $p$ for $\mathrm{N}=100$ (red, plus), 150(green, cross), 200(blue, star), 300 (magenta, square), 500(cyan, solid square), 600(black, circle), $\tau=0.35, \epsilon=0.01$. b) Normalized transition curves $p-p_{t} / \delta$ for different N. c) Variation of the scaling index with $1 / \mathrm{N}$ with error bar shown in red.

\section{E. Transitions to amplitude death in random networks with non-uniform probabilities of connections}

In the study presented in sections IIA-IID, the probability of connections for generating the random network $p$ is kept the same for slow and fast nodes. We now 
consider a much more heterogeneous case of random networks, generated with three different probabilities and study the effect of slow and fast dynamics on it. This is done by taking the probability with which a slow system connects to another slow system as $p_{1}$, while a fast system connects with another fast system with $p_{2}$ and a slow system connects with a fast system with $p_{3}$. We compute the fraction of realizations resulting in amplitude death in this random network of slow and fast systems by varying $p_{3}$ for different sets of values of $p_{1}$ and $p_{2}$.

It is interesting to note that amplitude death happens even in a bipartite network, with $p_{1}=0$ and $p_{2}=0$ but non-zero $p_{3}$. However, having non-zero values for $p_{1}$ and $p_{2}$ helps the network to reach amplitude death state at lower values of probability $p_{3}$ and the minimum $p_{3}$ for this transition becomes smaller with increasing $p_{1}$ and $p_{2}$ (Fig. 8). We also study the special cases when with
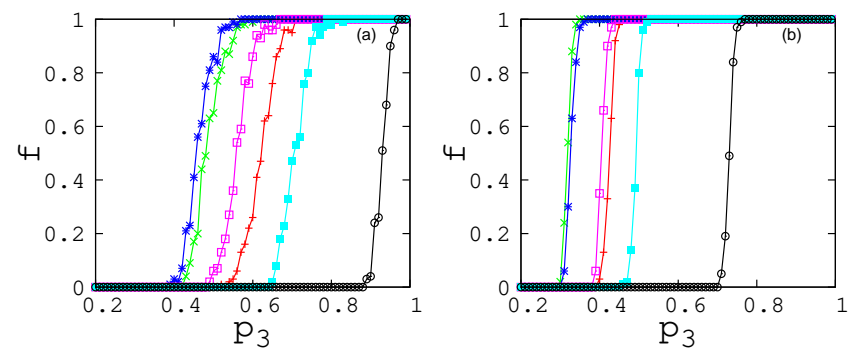

FIG. 8. (colour online)Fraction of realizations for transition to $\mathrm{AD}$ for random network of heterogeneous probabilities for varying $p_{3}$ a)p $\left.1=0, \mathrm{p} 2=0, \mathrm{~b}\right) \mathrm{p} 1=0.3, \mathrm{p} 2=0.3$. Here $\tau=0.35$, $\epsilon=0.01$. $m=30$ (red, plus), 40(green, cross), 50(blue, star), 60(magenta, square), 70(cyan, solid square), 80(black, circle).
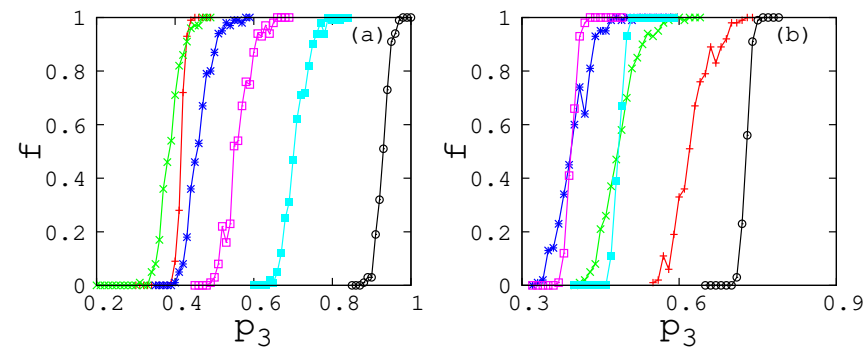

FIG. 9. (colour online)Fraction of realizations for transition to $\mathrm{AD}$ for a random network of heterogeneous probabilities for varying $p_{3}$ a)p1 $\left.1=0, \mathrm{p} 2=0.8, \mathrm{~b}\right) \mathrm{p} 1=0.8, \mathrm{p} 2=0$, keeping $\tau=0.35$, $\epsilon=0.01$. $m=30$ (red, plus), 40(green, cross), 50(blue, star), 60(magenta, square), 70(cyan, solid square), 80(black, circle).

$p_{1}=0, p_{3}$ is varied keeping $p_{2}$ fixed as well as $p_{2}=0$ and $p_{3}$ is varied with $p_{1}$ fixed. The results shown are for $p_{1}=0.8$ and $p_{2}=0.8$ in the respective cases and $p_{3}$ is varied (Fig. 9a,b).
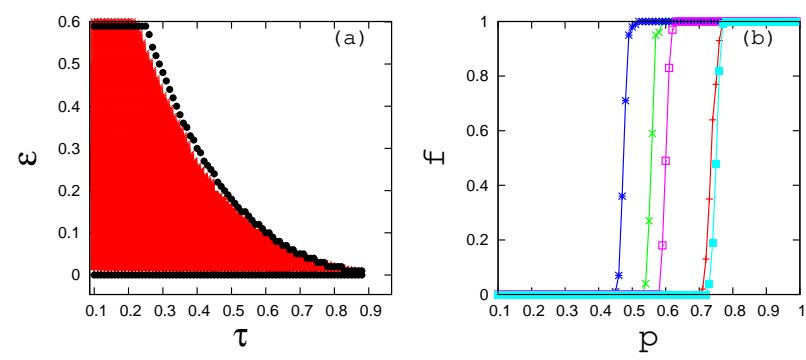

FIG. 10. (colour online)a)Region showing $\mathrm{AD}$ in $\tau, \epsilon$ plane for Landau-Stuart systems on a random network, $p=0.5$, $m=50, N=100, \mathrm{~b})$ variation of fraction of realizations with $p$ for $\tau=0.35, \epsilon=0.01$. $m=30$ (red), 40(green), 50(blue), 60(magenta), 70(cyan).

\section{F. Random network of Landau Stuart systems}

We do a similar study for slow and fast Landau Stuart oscillators on random networks given by the equations

$$
\begin{aligned}
& \dot{x}_{i}=\tau_{i}\left(\left(a-x_{i}{ }^{2}-y_{i}{ }^{2}\right) x-\omega y_{i}\right)+\tau_{i} \epsilon \sum_{j=1}^{N} A_{i j}\left(x_{j}-x_{i}\right) \\
& \dot{y}_{i}=\tau_{i}\left(\left(a-x_{i}{ }^{2}-y_{i}{ }^{2}\right) y+\omega x_{i}\right)
\end{aligned}
$$

The results are qualitatively similar for amplitude death and oscillatory behaviors. The region of $\mathrm{AD}$ is numerically calculated and shown for $p=0.5, m=50, \mathrm{~N}=100$ in $\tau, \epsilon(\operatorname{Fig}[10 \mathrm{a})$. Choosing the $\tau$ and $\epsilon$ from the region of $\mathrm{AD}$ one can show the variation for fraction of realizations of $\mathrm{AD}$ while $p$ varies. In this case also an optimum number of slow systems exists for which the transition occurs for minimum value of probability $p$ as shown in Fig $10 \mathrm{~b}$.

\section{Stability analysis for amplitude death state}

We find the onset of amplitude death is due to the stabilisation of the unstable fixed point at $(0,0)$. Hence the transition to $\mathrm{AD}$ can be obtained by a detailed stability analysis. We note that stability of AD state is analysed in the case of two coupled systems of differing time scales, globally coupled networks and minimal networks or motifs of 3 or 4 systems $\underline{44} \underline{47}$. In the present study, with heterogeneity in the pattern of connections also, AD occurs due to interplay of time scales and topology.

We can write Jacobian of $\mathrm{n}$-dimensional slow and fast systems on a network of size $\mathrm{N}$ as

$$
\mathbf{J}=(\boldsymbol{\tau} \cdot \mathbf{I}) \otimes \mathbf{F}^{\prime}+(\boldsymbol{\tau} \cdot \mathbf{L}) \otimes \mathbf{H}
$$

where $\boldsymbol{\tau}$ is an NxN matrix in which $\tau_{i j}$ corresponds to $\tau_{i}$ of eqn(11) for all $\mathrm{j}$. I is $\mathrm{NxN}$ identity matrix. Dot product $(\cdot)$ is defined here by the element wise product of two matrices, and cross product $(\otimes)$ is defined as each element of the former matrix being multiplied by the later matrix as a block ${ }^{63}$. $\mathbf{L}$ is the laplacian matrix,

$$
\mathbf{L}=\mathbf{D}-\mathbf{A}
$$


where $\mathbf{D}$ is the diagonal degree matrix of the network, and $\mathbf{A}$ is adjacency matrix. $\mathbf{F}^{\prime}$ is Jacobian of the intrinsic system around fixed point and is an nxn matrix, where $\mathrm{n}$ is the dimension of a single system on each node. $\mathbf{H}$ is nxn coupling matrix.

In the case of Landau-Stuart oscillators systems with coupling function as given in eqn(5) and $\mathrm{AD}$ at the fixed point $(0,0)$, we have

$$
\mathbf{F}^{\prime}=\left(\begin{array}{cc}
-a & -\omega \\
\omega & a
\end{array}\right), \mathbf{H}=\left(\begin{array}{cc}
\epsilon & 0 \\
0 & 0
\end{array}\right)
$$

We construct the matrix $\mathbf{J}$ following the equation (6) and calculate its eigenvalues. Scanning the whole plane of $\tau \epsilon$, we estimate the values of $\tau$ and $\epsilon$, where the real part of the largest eigenvalue crosses zero. The plot of these values in $(\tau, \epsilon)$ plane gives the transition curve to $\mathrm{AD}$ or the boundary for the stable region for AD. This is shown in black dots in Fig 10 . We note that the boundaries calculated using the above stability analysis agrees well those obtained by direct numericalGraph partitions and cluster synchronization in networks of oscillators simulations.

\section{SCALE FREE NETWORKS OF SLOW AND FAST SYSTEMS}

As is well known, scale-free networks are inherently more heterogeneous than random networks, with broad distribution of nodal degrees and a few nodes with very high degrees, called hubs. Hence the emergent dynamics due to interactions among slow and fast dynamical systems on such a scale free network will be interesting. For this we generate several realizations of scale free networks using Barabási-Albert algorithm ${ }^{64}$ and consider the dynamics on each node of the network, as that of periodic Rössler systems. The equations for the dynamics on such a network will be the same as eqn( 2) with all parameters taken the same way. But the adjacency matrix $A_{i j}$ is taken as per the scale free network topology obtained from the Barabási-Albert algorithm.

In a typical calculation, we take a network of size 100, with a set of $m$ nodes evolving at the slower time scale. Since in a scale free network, hubs play the role of control nodes, we mostly concentrate on cases where hubs follow slower dynamics. Hence in this case the number of slow systems required for AD to occur, is much smaller. Thus on a scale free network of 100 systems even with eight of the higher degree nodes or hubs having a time scale mismatch of $\tau$, we find the dynamics of all the systems can be suppressed to AD state (Fig 11). We isolate the region of $\mathrm{AD}$ in $(\tau, \epsilon)$ plane as the region where the difference between the global maxima and global minima of all the oscillators goes to zero. This is shown in Fig. 12 ,

We also estimate the minimum number of slow systems required to induce $\mathrm{AD}$ starting with the highest degree as slow and increasing the number one by one. For each

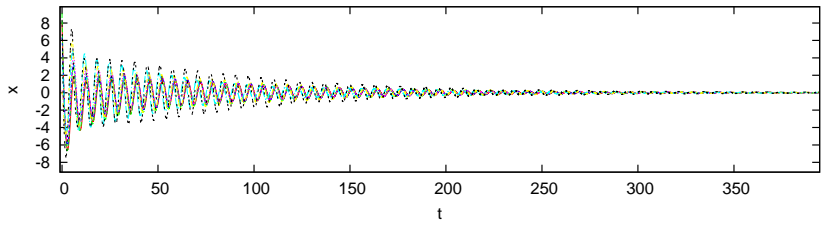

FIG. 11. (colour online) Time series of few typical x-variables are plotted, showing amplitude death in a scale free network of periodic Rössler systems for $\tau=0.3, \epsilon=0.2$ and $m=8$.

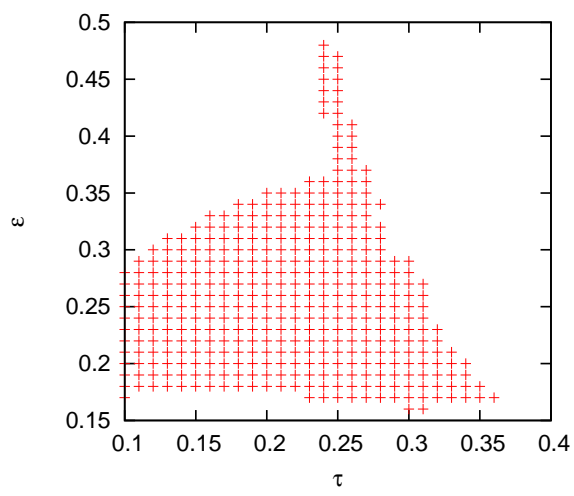

FIG. 12. (colour online) AD region in $(\tau, \epsilon)$ plane for 100 periodic Rössler systems coupled on a typical realization of scale free network with 8 hubs taken from the high degree end slow in dynamics.

case, the average amplitude differences of all the oscillators is calculated. The plot of this averaged amplitude $\left.\left(<A_{\text {diff }}\right\rangle\right)$ with the number of slow hubs $m$ gives this as the value of $m$ at which $\left(<A_{\text {diff }}>\right)$ becomes zero. This is repeated for different realizations and shown in Fig.13. Here the values of $\tau$ and $\epsilon$ are chosen from the $\mathrm{AD}$ region in Fig. 12 .

We repeat the above study using Landau-Stuart oscil-

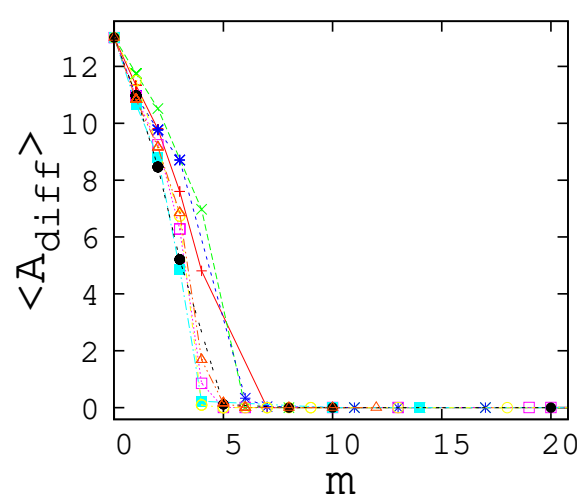

FIG. 13. (colour online) $\left\langle A_{\text {diff }}>\right.$ vs $m$ showing the minimum value for number of slow hubs required for $\mathrm{AD}$ to occur for periodic Rössler systems on a scale free network. Here $\tau=0.12$ and $\epsilon=0.25$ Different colors correspond to different realizations. 


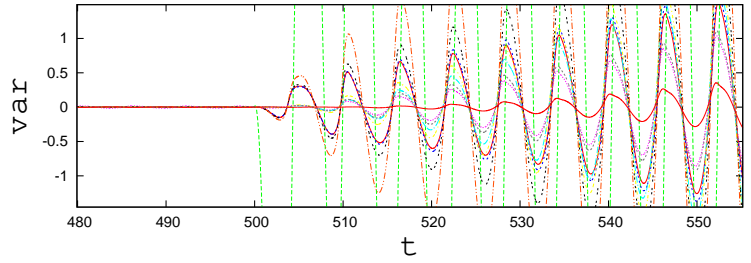

FIG. 14. (colour online)Variance of few typical periodic Rössler oscillators (var) with time to show that each oscillator takes a different time to move away from the synchronized state. $\tau=0.3, \epsilon=0.03$.

lator as nodal dynamics. We find qualitatively similar results for this case also with $\mathrm{AD}$ state and frequency synchronization.

\section{A. Spreading of slowness on scale free networks}

When there is no time scale mismatch in the dynamics of systems, all the systems on a scale free network, can be completely synchronized with a sufficiently strong coupling strength. Starting with such a state, after giving sufficient time so that all the oscillators settle to complete synchronization, we make one of the nodes, called source node, slower in its dynamics. Clearly this can disrupt the dynamics of all other nodes as the slowness spreads over the network. Consequently, all oscillators will then move away from the state of complete synchronization.

Due to the heterogeneity of connections in the scale free network, the time taken by each oscillator to move away from synchronization will not be the same. We analyze this scenario in terms of the degree of the node and shortest path from the source node, in the following two ways. We calculate the change in the variance of all oscillators in time. When they are completely synchronized, the variance would be zero as shown in Fig. 14 . When one node is made slow, the variation of each oscillator from the mean of anticipated synchronized oscillations(the synchronized oscillation they would have followed if this node was not made slow), is nonzero indicating onset of desynchronization. From the Fig. 14 it is evident that for each oscillator the time taken for the variance to go to a non zero value $\pm \nu$ is different, with the source node taking the least time obviously. This time, $t_{\nu}$, for each oscillator to reach a specific value $\pm \nu$ (typically -0.01 or 0.01 ) for its variation is plotted as a function of the degree of the nodes. It is easy to see that $t_{\nu}$ increases as the shortest path of that node from the source node increases. We repeat this for several nodes as sources, including hubs and low degree nodes. Fig. 15 shows the plot of $t_{\nu}$ against degree of nodes for the two cases with a hub as the source node and a low degree node as the source node for a typical realization. In the case where a hub is the source of slowness, we see most of the nodes move away in much shorter times since the shortest path from the source node is small.
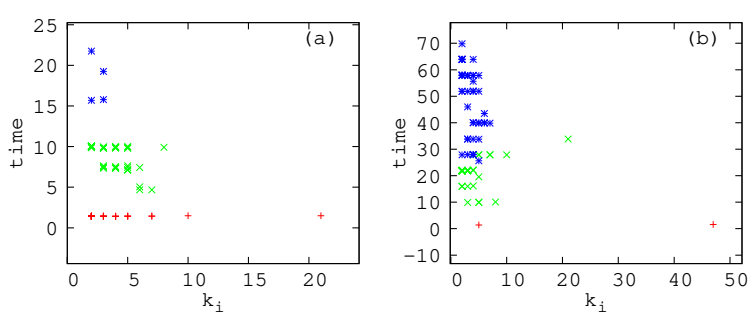

FIG. 15. (colour online)Time taken for each oscillator to move away from synchrony is plotted with its degree $\left(k_{i}\right)$ when one source node becomes slow. This is shown for a particular realisation of the network of 100 systems where a) the highest degree hub is made slow with degree 47 and in b) the lowest degree node is made slow with degree 2. Different colors represent different shortest path lengths from the source node with shortest path 1(red, plus), 2(green, cross), 3(blue, star). Here $\tau$ is 0.3 for the source node and $\epsilon$ is taken as 0.03 .
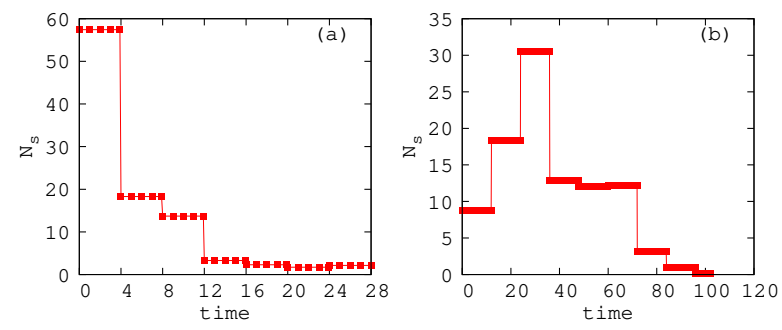

FIG. 16. (colour online) Number of systems that move away from synchrony $\left(N_{s}\right)$ in a range of time is plotted with time. The results shown are averaged over six realisations. Here a) corresponds to the highest hub as source of slowness for each realisation and $\mathrm{b}$ ) corresponds to the lowest degree node being made slow. In this case also, $\tau$ is 0.3 for the source node and $\epsilon=0.03$.

We repeat the study for different realizations of the network and calculate the number of systems that get desynchronized in a given time and this number averaged over the realizations varies as shown in Fig. 16, for the two cases, when a hub and a low degree node are the sources of slowness. It is interesting to note that the total time taken for all the systems to move away from each other decreases with the degree of the source node as a power law. This is clear from Fig. 17 shown for a typical realization of the network. We find that this curve can be fitted with a function as

$$
T(k)=(a / k)+b
$$

with $a=180$ and $b=18$. The desynchronization transition is followed by a reorganization of the whole network.

\section{B. Self organization of the network to frequency synchronized state}

Once synchrony is disturbed as discussed above due to a single node going slow, de-synchronization sets in 


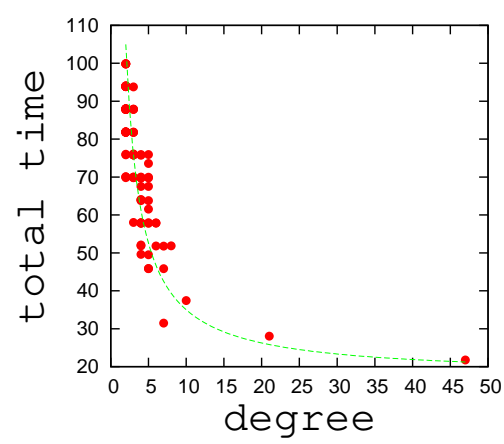

FIG. 17. (colour online)Total time taken for all oscillators in the network to move away from synchrony is plotted against the degree of source node for periodic Rössler systems on scale free network. The source node has time scale as $\tau=0.3$ and $\epsilon=0.03$.

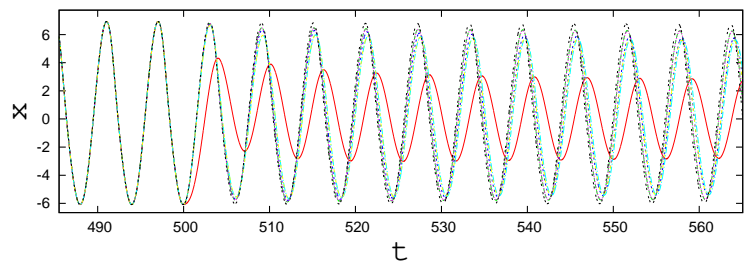

FIG. 18. (colour online)Time series of few typical x-variables of periodic Rössler systems are plotted to show the frequency synchronized state reached from the state of complete synchronization after one node is made slow. Here $\tau=0.3$ for the slow node and $\epsilon=0.1$.

characteristic times depending on the degrees of nodes in the network. Subsequent to this, given sufficient time, all the oscillators are found to reorganize themselves into a frequency synchronized state. (Fig. (18). This is an interesting and novel phenomenon of self-organization, where the network goes from a collective behavior of complete synchronization to another less ordered but coherent emergent state of frequency synchronization by re-adjusting the dynamics of all the nodes, after the network is perturbed by making one node slow.

To characterize this process, we calculate the frequency of each oscillator using eqn 3 and plot them with time(Fig [19 $)$. The figure shows the synchronized frequencies in the beginning, the de-synchronized frequencies just after one node is made slow at $\mathrm{t}=500$, and finally the re-adjusted lower frequency after self-organization to frequency synchronized state. We also study the time taken for self-organization, called self organization time, $\left(t_{\text {so }}\right)$, averaged over several realizations for $\tau$ varying in the range $0.2-0.8$ for a particular $\epsilon=0.1$, (Fig $19 \mathrm{~b}$ ). We observe that $t_{s o}$ decreases with the increase in the time scale mismatch introduced on the source node, along a straight line.
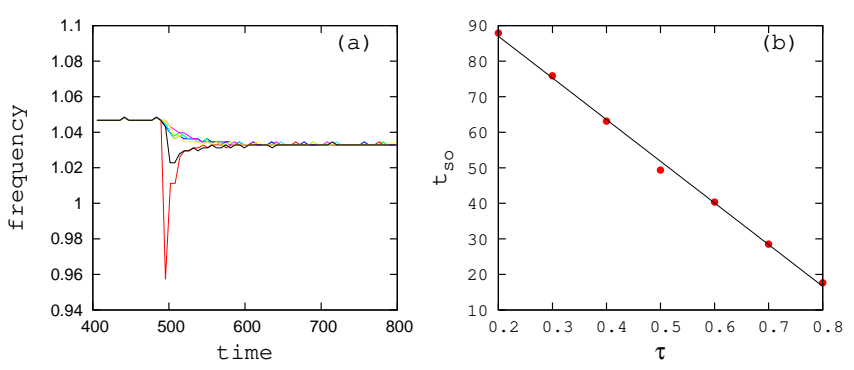

FIG. 19. (colour online)a) Self-organization of oscillators into frequency synchronized state for the whole network for a typical realization of scale free network after one hub is made slow. On the $\mathrm{x}$ axis the time and on the $\mathrm{y}$ axis the corresponding frequency are shown. At time $=500$ one hub is made slow, b) Time taken to organize into the new synchronized state, $t_{s o}$, averaged over six realizations is plotted for different $\tau$ values with $\epsilon$ kept at 0.1. $t_{\text {so }}$ falls off with increasing mismatch $\tau$ as a straight line.

\section{Scale free network with multiple time scales}

In this section, we study the collective dynamics of nonlinear systems on a scale free network where the time scale of each node varies with its degree following the relation

$$
\tau_{i}=2 / k_{i}
$$

This is chosen such that the node with highest degree will have the slowest time scale and the time scale increases as degree decreases. Since the network is scale free, the number of different degrees, $k_{i}$ and hence the number of different time scales, $\tau_{i}$, will be less than $\mathrm{N}$ but still will have a multiplicity of time scales. Thus, for one typical realisation of 1000 nodes, we get 30 different time scales in the network. We find the presence of multiple time scales, forces the whole network to collapse to a state of $\mathrm{AD}$. The onset of $\mathrm{AD}$, after a threshold coupling strength, is evident from the plot of $\left\langle A_{d i f f}\right\rangle$, for different values of $\epsilon$, in Fig. 20 with three different sizes $\mathrm{N}=100,500$ and 1000 .

For lower values of $\epsilon$, prior to onset of $\mathrm{AD}$, we see oscillations with differing amplitudes. But even with multiple time scales, the connectivity through the network makes the systems organize into three groups, high degree nodes with lower time scales having smaller amplitudes, low degree nodes with faster time scales having larger amplitudes and an intermediate group with amplitudes in between. This is clear from the distribution of amplitudes of all the oscillators in the network from six realizations of the network of size $\mathrm{N}=1000$ (Fig 21).

Similarly we do the analysis for another distribution of time scales on nodal dynamics of scale free network. In this case we choose the $i^{\text {th }}$ node to follow a time scale $\tau_{i}$ drawn from a normal distribution with mean 0.5 and standard deviation 0.15. Since in this case there is no correlation between the degree of the node and its time scales and the network is hierarchical, we take multiple 


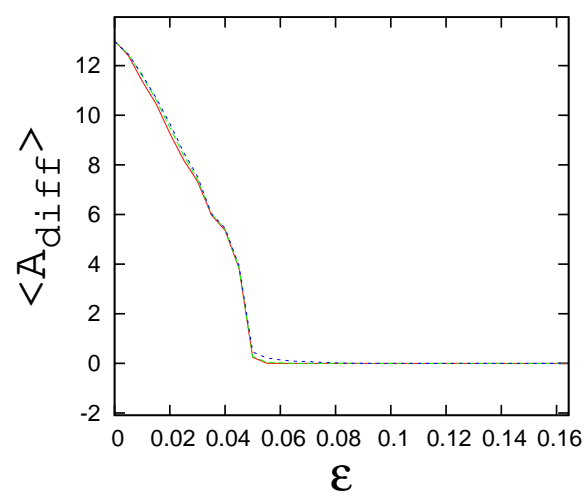

FIG. 20. (colour online) $<A_{\text {diff }}>$ vs $\epsilon$ in a typical realization of scale free network showing onset of AD due to multiplicity of time scales for periodic Rössler systems with $\tau_{i}=2 / k_{i}$ for $\mathrm{N}=100$ (red), 500(green), 1000(blue).

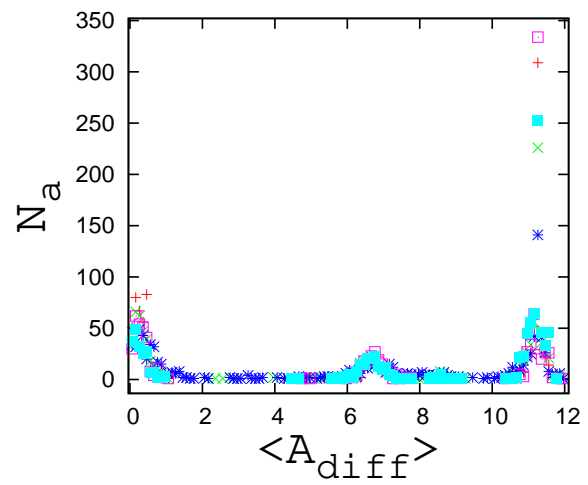

FIG. 21. (colour online)Distribution of amplitudes of 1000 periodic Rössler systems with multiple time scales on a scale free network for $\epsilon=0.03$, with the time scales distributed as eqn. 9. The different colours indicate different realizations of the network.

realizations of the distribution and analyse the dynamics. The onset of $\mathrm{AD}$ state on a scale free network of periodic Rössler systems and the threshold value of $\epsilon$ is shown in (Fig. 22 a). We also estimate the transition probability as the fraction of realizations of time scale distributions for which $\mathrm{AD}$ occurs, for different values of $\epsilon$ and plot this fraction (f) with $\epsilon$ to see the transition curve to $\mathrm{AD}$. (Fig. 22b)

\section{CONCLUSION}

The study reported, in general, addresses the important question of how the mismatch in dynamical time scales of different interacting units can affect the collective performance of a complex system. We consider nonlinear dynamical systems with complex interaction patterns modelled by random and scale free topologies. We are motivated by the fact that one important factor for heterogeneity of interacting systems in real-world com-
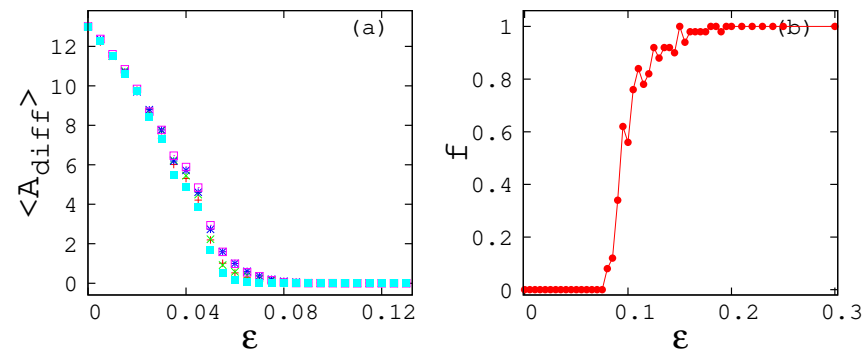

FIG. 22. (colour online) Onset of $\mathrm{AD}$ on a scale free network of 100 periodic Rössler systems with normal distribution for time scales. a) $<A_{\text {diff }}>$ vs $\epsilon$ in 5 typical realizations (shown in different colour) of time scale distributions showing onset of $\mathrm{AD}$. b)Fraction of realizations of time scale distributions showing transition to $\mathrm{AD}$ with $\epsilon$ for 50 realizations.

plex systems is the diversity in time scales. This makes our study highly relevant in understanding such systems from a dynamical system's perspective.

We study the robustness of coupled oscillator networks which are widely used as models for understanding the dynamics of networked systems in biology, physics, and engineering and see how increasing the heterogeneity in time scales makes them undergo transitions in their dynamics. The emergent dynamics is then characterized in terms of the average amplitude of oscillations and the common frequency of the coupled systems.

The results presented are primarily for periodic Rössler systems, with two distinct time scales. However qualitatively similar results are obtained in the case of random networks, with Landau-Stuart oscillators, chaotic Rössler and Lorenz systems.

When the network of connections is random, we study suppression and recovery of oscillations and cross over in amplitudes and frequency as number of slow systems increases. The transition to AD in terms of the probability of connections $\mathrm{p}$ is found to scale with network size, the index of scaling being $2 / 3$.

If the systems are connected on a scale free network with heterogeneity in the degrees, we find hubs can function as control nodes in the emergent dynamics. We study the spread of slowness through the network due to one hub being slow and discuss the self-organization of the whole network from completely synchronized state to a state of frequency synchronization. The hierarchical structure of this network leads to different patterns in the spread of slowness due to one slow source node. The study of the times involved gives an estimate of minimum time within which corrective measures are to be initiated to restore the network dynamics.

We also observe transition to AD on a scale free network, with multiple time scales, with time scale of each node depending on its degree and with time scales drawn from a normal distribution.

In all cases studied, suppression of dynamics with the whole network settling to a common fixed point, seems to be the most prevalent emergent state. We note that 
amplitude death due to time scale diversity has been reported in a few specific cases with global coupling 44.45 . Our study establishes how difference in dynamical time scales of systems induces AD in systems, interacting with random and scale free patterns of connections. So also we show recovery of oscillations of any desired common frequency is possible by tuning the time scales of the systems. It is interesting that cooperative phenomenon of lower order like frequency synchronization, may still emerge even in the presence of time scale diversity. As reported recently in modular networks of neurons $\frac{65}{5}$, tuning the time scales can also result in a variety of specific activity patterns with spatial order and frequency locking.

${ }^{1}$ Ogawa, Seiji, et al. "An approach to probe some neural systems interaction by functional MRI at neural time scale down to milliseconds." Proceedings of the National Academy of Sciences 97.20 (2000): 11026-11031.

${ }^{2}$ Bullmore, Ed, and Olaf Sporns. "Complex brain networks: graph theoretical analysis of structural and functional systems." Nature Reviews Neuroscience 10.3 (2009): 186.

${ }^{3}$ Boyden, Edward S., et al. "Millisecond-timescale, genetically targeted optical control of neural activity." Nature Neuroscience 8.9 (2005): 1263.

${ }^{4}$ Gilman, A. G. "Guanine nucleotide-binding regulatory proteins and dual control of adenylate cyclase." The Journal of clinical investigation 73.1 (1984): 1-4.

${ }^{5}$ Radovick, Sally, et al. "A mutation in the POU-homeodomain of Pit-1 responsible for combined pituitary hormone deficiency." Science 257.5073 (1992): 1115-1118.

${ }^{6}$ Goldberger, Ary L., C-K. Peng, and Lewis A. Lipsitz. "What is physiologic complexity and how does it change with aging and disease?." Neurobiology of aging 23.1 (2002): 23-26.

${ }^{7}$ Rivera, Victor M., et al. "Regulation of protein secretion through controlled aggregation in the endoplasmic reticulum." Science 287.5454 (2000): 826-830.

${ }^{8}$ Das, D., and D. S. Ray. "Multiple time scale based reduction scheme for nonlinear chemical dynamics." The European Physical Journal Special Topics 222.3-4 (2013): 785-798.

${ }^{9}$ Andrews, Steven S., and Dennis Bray. "Stochastic simulation of chemical reactions with spatial resolution and single molecule detail." Physical biology 1.3 (2004): 137.

${ }^{10}$ Carter, E. A., et al. "Constrained reaction coordinate dynamics for the simulation of rare events." Chemical Physics Letters 156.5 (1989): 472-477.

${ }^{11}$ Schiestel, Roland. "Multiple time scale modeling of turbulent flows in onepoint closures." The Physics of fluids 30.3 (1987): 722-731.

${ }^{12}$ Hanjalic, K., B. E. Launder, and R. Schiestel. "Multiple-timescale concepts in turbulent transport modeling." Turbulent Shear Flows 2 (1980): 36-49.

${ }^{13} \mathrm{Kim}, \mathrm{S}-\mathrm{W}$., and C-P. Chen. "A multiple-time-scale turbulence model based on variable partitioning of the turbulent kinetic energy spectrum." Numerical Heat Transfer, Part B Fundamentals 16.2 (1990): 193-211.

${ }^{14}$ Honey, Christopher J., et al. "Network structure of cerebral cortex shapes functional connectivity on multiple time scales." Proceedings of the National Academy of Sciences 104.24 (2007): 10240-10245.

${ }^{15}$ Persson, Erik, and Bertil Halle. "Cell water dynamics on multiple time scales." Proceedings of the National Academy of Sciences 105.17 (2008): 6266-6271.

${ }^{16}$ Henzler-Wildman, Katherine A., et al. "A hierarchy of timescales in protein dynamics is linked to enzyme catalysis." Nature 450.7171 (2007): 913.

${ }^{17}$ Gilboa, Gail, Ronen Chen, and Naama Brenner. "History- dependent multiple-time-scale dynamics in a single-neuron model." Journal of Neuroscience 25.28 (2005): 6479-6489.

${ }^{18}$ Alex Arenas, Albert Daz-Guilera, Jürgen Kurths, Yamir Moreno, and Changsong Zhou. "Synchronization in complex networks." Physics reports, 469(3):93-153, 2008.

${ }^{19}$ Stefano Boccaletti, Jürgen Kurths, Grigory Osipov, DL Valladares, and CS Zhou. "The synchronization of chaotic systems." Physics reports, 366(1-2):1-101, 2002.

${ }^{20}$ Meng Zhan, Xingang Wang, Xiaofeng Gong, GW Wei, and CH Lai. "Complete synchronization and generalized synchronization of one-way coupled time-delay systems." Physical Review E, 68(3):036208, 2003.

${ }^{21}$ Jianquan Lu and Jinde Cao. "Adaptive complete synchronization of two identical or different chaotic (hyperchaotic) systems with fully unknown parameters." Chaos: An Interdisciplinary Journal of Nonlinear Science, 15(4):043901, 2005.

${ }^{22}$ C.A.S Batista, A.M Batista, J.A.C De Pontes, R.L Viana, and S.R Lopes. "Chaotic phase synchronization in scale-free networks of bursting neurons." Physical Review E, 76(1):016218, 2007.

${ }^{23}$ Sarika Jalan and Aradhana Singh, "Cluster synchronization in multiplex networks", Europhysics Letters, 113, 3, (2016)

${ }^{24}$ Sarika Jalan and Aradhana Singh, "Impact of heterogeneous delays on cluster synchronization", Phys. Rev. E 90, 042907 (2014)

${ }^{25}$ Motter, A. E., Myers, S. A., Anghel, M. and Nishikawa, T. "Spontaneous synchrony in power-grid networks." Nature Phys. 9, 191197 (2013).

${ }^{26}$ Levy, Ron, et al. "High-frequency synchronization of neuronal activity in the subthalamic nucleus of parkinsonian patients with limb tremor." Journal of Neuroscience 20.20 (2000): 7766-7775.

${ }^{27}$ Fries, Pascal, et al. "Modulation of oscillatory neuronal synchronization by selective visual attention." Science 291.5508 (2001): $1560-1563$

${ }^{28}$ Szabadics, János, Andrea Lorincz, and G, Tamás. "Beta and gamma frequency synchronization by dendritic gabaergic synapses and gap junctions in a network of cortical interneurons." Journal of Neuroscience 21.15 (2001): 5824-5831.

${ }^{29}$ Renato E Mirollo and Steven H Strogatz. "Amplitude death in an array of limit-cycle oscillators." Journal of Statistical Physics, 60(1-2):245-262, 1990.

${ }^{30}$ Garima Saxena, Awadhesh Prasad, and Ram Ramaswamy. "Amplitude death: The emergence of stationarity in coupled nonlinear systems." Physics Reports, 521(5):205-228, 2012.

${ }^{31}$ Awadhesh Prasad, Mukeshwar Dhamala, Bhim Mani Adhikari, and Ramakrishna Ramaswamy. "Amplitude death in nonlinear oscillators with nonlinear coupling." Physical Review E, 81(2):027201, 2010.

${ }^{32}$ DV Ramana Reddy, Abhijit Sen, and George L Johnston. "Time delay induced death in coupled limit cycle oscillators." Physical Review Letters, 80(23):5109, 1998.

${ }^{33}$ Rajat Karnatak, Ram Ramaswamy, and Awadhesh Prasad. "Amplitude death in the absence of time delays in identical coupled oscillators." Physical Review E, 76(3):035201, 2007.

${ }^{34}$ V. Resmi, G Ambika, and R. E Amritkar. "General mechanism for amplitude death in coupled systems." Physical Review E, 84(4):046212, 2011.

${ }^{35}$ V. Resmi, G Ambika, R. E Amritkar, and G. Rangarajan. "Amplitude death in complex networks induced by environment". Physical Review E, 85(4):046211, 2012.

${ }^{36}$ X. M. Zhang, Y. C. Wu, and J. H. Peng, "Analytical conditions for amplitude death induced by conjugate variable couplings" Int. J. Bifurcation Chaos 21, 225 (2011).

${ }^{37}$ N. N. Zhao, Z. K. Sun, X. L. Yang, and W. Xu, "Explosive death of conjugate coupled Van der Pol oscillators on networks", Phys. Rev. E 97, 062203 (2018).

${ }^{38}$ A. Sharma, P. R. Sharma, and M. D. Shrimali, "Amplitude death in nonlinear oscillators with indirect coupling", Phys. Lett. A 376, 1562 (2012).

${ }^{39}$ N. K. Kamal, P. R. Sharma, and M. D. Shrimali, "Oscillation suppression in indirectly coupled limit cycle oscillators", Phys. Rev. E 92, 022928 (2015). 
${ }^{40}$ A. Sharma and M. D. Shrimali, "Amplitude death with meanfield diffusion", Phys. Rev. E 85, 057204 (2012).

${ }^{41}$ T. Banerjee and D. Ghosh, "Transition from amplitude to oscillation death under mean-field diffusive coupling", Phys. Rev. E 89, 052912 (2014).

${ }^{42} \mathrm{~T}$. Banerjee and D. Ghosh, "Experimental observation of a transition from amplitude to oscillation death in coupled oscillators", Phys. Rev. E 89, 062902 (2014).

${ }^{43}$ T. Banerjee, D. Biswas, D. Ghosh, B. Bandyopadhyay, and Jürgen Kurths, "Transition from homogeneous to inhomogeneous limit cycles: Effect of local filtering in coupled oscillators", Phys. Rev. E 97, 042218 (2018).

${ }^{44}$ Silvia De Monte, Francesco dOvidio, and Erik Mosekilde, "Coherent Regimes of Globally Coupled Dynamical Systems", Phys. Rev. Lett. 90, 054102 (2003)

${ }^{45}$ Wei Zou, Meng Zhan, and Jürgen Kurths, "Amplitude death in globally coupled oscillators with time-scale diversity", Phys. Rev. E 98, 062209 (2018)

${ }^{46}$ Kajari Gupta and G Ambika, "Suppression of dynamics and frequency synchronization in coupled slow and fast dynamical systems", Eur Phy J B 89(6), 1-8(2016)

${ }^{47}$ Kajari Gupta and G Ambika, "Dynamics of slow and fast systems on complex networks", Indian Academy of Sciences Conference Series 1:1 (2017)

${ }^{48}$ P. J. Menck, J. Heitzig, Jürgen Kurths and H. J. Schellnhuber, "How dead ends undermine power grid stability." Nat. Commun. 5, 3969 (2014).

${ }^{49}$ Jenkins, A. "Self-oscillation." Phys. Rep. 525, 167-222 (2013).

${ }^{50}$ Tchumatchenko, T. and Clopath, C. "Oscillations emerging from noise-driven steady state in networks with electrical synapses and subthreshold resonance." Nat. Commun. 5, 5512 (2014).

${ }^{51}$ Morino, K., Tanaka, G. and Aihara, K. "Efficient recovery of dynamic behavior in coupled oscillator networks." Phys. Rev. E 88, 032909 (2013).

${ }^{52}$ A. Majdandzic et al. "Spontaneous recovery in dynamical networks." Nat. Phys. 10, 3438 (2013).

${ }^{53}$ W. Zou, , C. Yao and M. Zhan, "Eliminating delay-induced oscillation death by gradient coupling." Phys. Rev. E 82, 056203 (2010).

${ }^{54}$ W. Zou, D. V. Senthilkumar, M. Zhan and Jürgen Kurths, "Re- viving oscillations in coupled nonlinear oscillators." Phys. Rev. Lett. 111, 014101 (2013).

${ }^{55}$ Wei Zou, D.V. Senthilkumar, Raphael Nagao, Istva n Z. Kiss, Yang Tang, Aneta Koseska, Jinqiao Duan and Jürgen Kurths, "Restoration of rhythmicity in diffusively coupled dynamical networks", Nature Communications 6, 7709 (2015)

${ }^{56} \mathrm{O}$ 'Neill, Robert V. "Recovery in complex ecosystems." Journal of Aquatic Ecosystem Stress and Recovery 6.3 (1998): 181-187.

${ }^{57}$ Goh, B. S. "Stability, vulnerability and persistence of complex ecosystems." Ecological Modelling 1.2 (1975): 105-116.

${ }^{58}$ Grimm, Nancy B., et al. "Integrated approaches to long-term studies of urban ecological systems: Urban ecological systems present multiple challenges to ecologists pervasive human impact and extreme heterogeneity of cities, and the need to integrate social and ecological approaches, concepts, and theory." AIBS Bulletin 50.7 (2000): 571-584.

${ }^{59}$ Cameron, Lynne, and Diane Larsen-Freeman. "Complex systems and applied linguistics." International journal of applied linguistics 17.2 (2007): 226-239.

${ }^{60}$ Yamashita, Yuichi, and Jun Tani. "Emergence of functional hierarchy in a multiple timescale neural network model: a humanoid robot experiment." PLoS computational biology 4.11 (2008): e1000220.

${ }^{61}$ Jamshidi, Neema, and Bernhard . Palsson. "Top-down analysis of temporal hierarchy in biochemical reaction networks." PLoS computational biology 4.9 (2008): e1000177.

${ }^{62}$ Michael T. Schaub, Neave O'Clery, Yazan N. Billeh, Jean-Charles Delvenne, Renaud Lambiotte and Mauricio Barahona, "Graph partitions and cluster synchronization in networks of oscillators", Chaos 26, 094821 (2016)

${ }^{63}$ Louis M. Pecora and Thomas L. Carroll, "Master stability function for synchronized coupled systems", Phys. Rev. Lett. 80, 10(1998)

${ }^{64}$ Réka Albert and Albert-Lszl Barabsi. "Statistical mechanics of complex networks. Reviews of modern physics", 74(1):47, 2002.

${ }^{65}$ Kunal Mozumdar, G. Ambika, "Frequency locking and travelling burst sequences in community structured network of inhibitory neurons with differing time-scales", Commun Nonlinear Sci Numer Simulat 69 320328(2019) 